\title{
Nuclear medicine imaging of neuroendocrine
}

\section{tumours}

Adil Al-Nahhas, chief of service of nuclear medicine, Department of Nuclear Medicine, Hammersmith Hospital, Imperial College

NHS Trust, London

Neuroendocrine tumours (NETs) are a clinically diverse group of tumours which commonly affect the gastroenteropancreatic tract and lungs. They are rare, with an incidence of $2-5 / 100,000$, but this figure is rising due to improvement in biochemical assays. NETs comprise carcinoid tumours and various pancreatic tumours such as gastrinoma, insulinoma, VIPoma, glucagonoma and somatostatinoma. Tumours may be benign or malignant and up to twothirds secrete hormones and tumour-specific markers such as chromogranin A, 5-hydroxyindoleaceticacid, gastrin, serotonin and neurokinin A. Prognosis is highly variable, with five-year survival ranging from $19 \%$ in metastatic disease to $93 \%$ in local disease. ${ }^{1}$ NETs are of interest due to their expression of cell membrane receptors that contribute to their detection and treatment. A minority of NETs are associated with genetic and hereditary syndromes such as multiple endocrine neoplasia type 1 .

The diagnosis of NETs has been vastly improved by increased efficiency and speed of relevant biochemical screening tests, making it possible to diagnose patients at an early stage of the disease. However, the accurate localisation required for surgical planning, follow-up and assessment of response to therapy relies on a combination of anatomical and functional imaging.

\section{Conventional imaging}

Conventional anatomical imaging is usually undertaken as part of the initial workup of patients with NETs which may include a combination of:

- ultrasound (US)

- computed tomography (CT)

- magnetic resonance imaging (MRI)

- angiography

- endoscopic retrograde cholangio-pancreatography (ERCP).
US scanning is useful but highly userdependent, with reduced sensitivity due to interference of bowel gas. Endoscopic US and ERCP can overcome this limitation but are relatively invasive. Contrastenhanced CT and MRI are reliable techniques for assessing pancreatic tumours, but both procedures have suboptimal sensitivity in the diagnosis and staging of NETs due to the small tumour size and multiple disease sites characterising this group of tumours.

A major drawback in assessing treatment response using cross-sectional imaging is the inability to assess metabolic viability within an anatomical residual mass. Angiography is useful in the detection of small islet cell tumours by selective arterial catheterisation and portal venous sampling, but is an invasive procedure. By comparison, functional imaging that targets receptor binding of radiolabelled peptides has shown high sensitivity and specificity, facilitates whole body imaging, and can be used to assess disease activity and response to therapy.

\section{Functional imaging of neuroendocrine tumours}

Functional imaging of NETs exploits the fact that the vast majority express somatostatin receptors on their cell membrane. Somatostatin is a cyclic hormone expressed in the central and peripheral nervous system, inhibiting the release of hormones such as glucagon and insulin by binding to G-coupled somatostatin receptors. ${ }^{2}$ Five human somatostatin receptors (hSSTR1-5) have been identified, of which hSSTR2 is the most commonly expressed on NET cell membranes.

A number of synthetic peptides such as octreotide have somatostatin agonist activity through the mechanism of receptor binding and are used to treat NETs. In the early 1990s, a radiolabelled version of octreotide, indium-111-DTPA-DPhe1octreotide ( ${ }^{111}$ In-octreotide), which has a high affinity for hSSTR2 and hSSTR5, was introduced for somatostatin receptor 
imaging (SSRI). ${ }^{3,4}{ }^{111} \mathrm{In}$-octreotide is injected intravenously (iv) and whole-body images obtained four and 24 hours later, with additional single photon emission tomography (SPECT) over areas of interest. Physiological uptake is seen in the spleen, kidneys and liver, with minimal uptake in pituitary and thyroid glands (Fig 1). The bowel and gallbladder may accumulate tracer in late images and are major pitfalls in the search for gut primary or metastatic liver lesions.

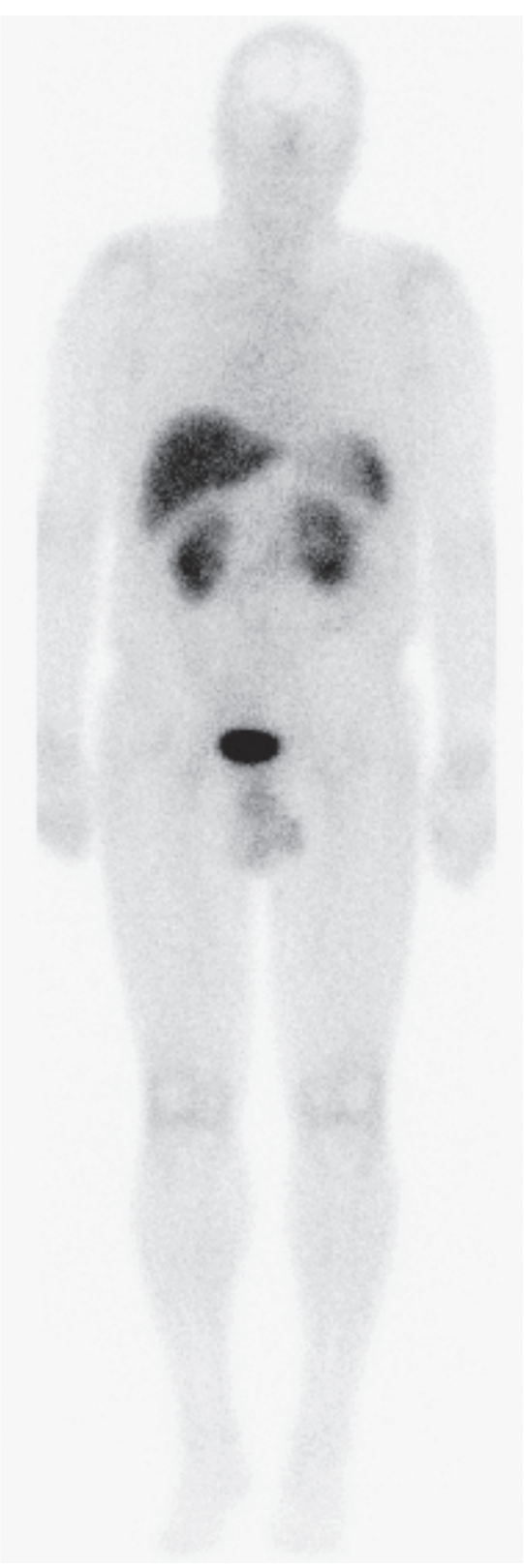

Fig 1. Anterior whole-body image of ${ }^{111}$ In-octreotide, with normal physiological uptake in the spleen, liver, kidneys and urinary bladder.
The largest body of experience with ${ }^{111} \mathrm{In}$ octreotide is from the Rotterdam group which performed SSRI in over 1,000 patients with NETs. They demonstrated $100 \%$ sensitivity for gastrinoma and glucagonoma, $96 \%$ for carcinoid and $69 \%$ for insulinoma. ${ }^{5}$ Subsequently, ${ }^{111}$ In-octreotide was established as the imaging method of choice for diagnosis and follow-up of NETs, aided by parallel developments in gamma camera hardware and a wider use of SPECT to improve image resolution.

Inevitably, cumulative experience of imaging with ${ }^{111}$ In-octreotide showed several drawbacks adversely affecting its diagnostic accuracy. Despite the universal use of SPECT, image resolution was not adequate to pick up lesions smaller than $1 \mathrm{~cm}$ due to the inherent physical qualities of ${ }^{111}$ In. This, coupled with the realisation that the affinity of octreotide to hSSTR2 is limited, means that lesions adjacent to areas of physiological uptake such as the spleen and kidneys are difficult to detect. In addition, the two-day imaging protocol is inconvenient for both patients and doctors.

An alternative radiopharmaceutical, iodine-123 metaiodobenzylguanidine $\left({ }^{123} \mathrm{I}-\mathrm{mIBG}\right)$ is also used in the detection of gastroenteric NETs that express the noradrenaline transporter. In addition to the imaging drawbacks listed above, however, it exhibits a lower sensitivity than ${ }^{111}$ In-octreotide (49\% vs $\left.91 \%\right)^{6}$ and uptake may be compromised by drug interference.

\section{PET/CT imaging of neuroendocrine tumours}

To overcome the limitations of SPECT radiopharmaceuticals, radionuclide NET imaging is now undertaken by hybrid positron emission tomography (PET)/CT which offers higher spatial resolution and better anatomical localisation. PET/CT imaging has revolutionised the management of cancer by improving resolution to approximately $5 \mathrm{~mm}$, allowing detection of small lesions and by fusing functional data with simultaneous CT for accurate localisation. PET/CT is currently an essential diagnostic and follow-up tool for a vast number of cancers, particularly lymphoma, melanoma, lung and colorectal cancer.

The first PET radiopharmaceutical to be used for detecting NETs was fluorine-18 fluorodeoxyglucose ( $\left.{ }^{18} \mathrm{~F}-\mathrm{FDG}\right)$, which accumulates in lesions with high metabolic rates and glucose utilisation. ${ }^{18} \mathrm{~F}-\mathrm{FDG}$ was found to be useful in detecting NETs ${ }^{7}$ but is limited to the minority of tumours that are undifferentiated and behave aggressively. Several studies have shown that ${ }^{18} \mathrm{~F}-\mathrm{FDG}$ is more sensitive than ${ }^{111}$ In-octreotide in the detection of poorly differentiated NETs but less sensitive for well-differentiated tumours. $^{8}$

Fluorine-18 -L-3,4-dihydroxypheny lalanine $\left({ }^{18} \mathrm{~F}-\mathrm{DOPA}\right)$ has been used in NET, based on its biochemical pathway in dopamine synthesis, uptake being proportional to tumour cell metabolism. Hoegerle et al found a high $(65 \%)$ sensitivity for

\section{Key points}

Neuroendocrine tumours (NETs) are slow growing but potentially malignant, increasing in incidence due to advances in hormone and tumour markers assays improving detection

Conventional imaging is routinely used for localisation but sensitivity is suboptimal due both to small size and spread of lesions

Functional imaging with indium-111-octreotide has better sensitivity than conventional imaging and detects increased expression of somatostatin receptors by tumours

New peptides with higher detection rates, labelled to positron emitting radionuclides, have been introduced for PET/CT imaging

Gallium-68-peptides PET/CT is the new gold standard in imaging NETs with sensitivity and specificity well beyond $90 \%$

KEYWORDS: gallium-68-Dotatate, indium-111-octreotide, neuroendocrine tumours, $\mathrm{PET} / \mathrm{CT}$ imaging, somatostatin receptors 
${ }^{18} \mathrm{~F}-\mathrm{DOPA}$ in detecting NETs compared with $29 \%$ sensitivity for ${ }^{18} \mathrm{~F}-\mathrm{FDG}$ and $57 \%$ sensitivity for ${ }^{111}$ In- octreotide. However, ${ }^{18} \mathrm{~F}-\mathrm{DOPA}$ has a major drawback of physiological uptake in the pancreas, making it less sensitive for the detection of small pancreatic lesions. It is also difficult and expensive to produce, and remains a research tool in the UK.

Carbon-11-hydroxytryptophan $\left({ }^{11} \mathrm{C}-5\right.$ HTP) targets the serotonin production pathway ${ }^{10}$ and was shown to be superior to ${ }^{111}$ In-octreotide, CT and ${ }^{18} \mathrm{~F}$-DOPA in the detection of NETs. Unfortunately, the short half-life of ${ }^{11} \mathrm{C}$ (20 min) restricts its use to facilities with an on-site cyclotron for radioisotope production.

\section{PET/CT with gallium-68 peptides}

The success in imaging NETs with PET/CT reached its climax with the introduction of gallium-68-peptides in 2001. ${ }^{11}$ The two major factors contributing to this breakthrough were the development of a gallium-68 $\left({ }^{68} \mathrm{Ga}\right)$ generator and the introduction of new somatostatin agonist peptides with very high affinity to hSSTR. The small size of the ${ }^{68} \mathrm{Ga}$ generator eliminates the need for a costly cyclotron facility, while its long half-life of 270 days ensures a daily supply of ${ }^{68} \mathrm{Ga}$ for at least one year. This provided an opportunity for departments with standard radiopharmacies to exploit this advanced imaging approach. ${ }^{12}$

Different ${ }^{68} \mathrm{Ga}$-labelled peptides $\left({ }^{68} \mathrm{Ga}\right.$ Dotatate, ${ }^{68} \mathrm{Ga}$-Dotatoc and ${ }^{68} \mathrm{Ga}$-Dotanoc) were soon introduced into clinical practice and proved to have very similar efficacies. The images are of high clarity and sensitivity due to early washout of surplus peptides from the body, allowing a short imaging time of about $25 \mathrm{~min}$. NETs that are easily missed or appear equivocal on ${ }^{111}$ In-octreotide show clearly on ${ }^{68} \mathrm{Ga}$-Dotatate (Fig 2). In a study by Gabriel et $a l^{13}$ on 84 patients with NETs comparing ${ }^{68} \mathrm{Ga}$-Dotatoc, ${ }^{111} \mathrm{In}$-octreotide and CT, ${ }^{68} \mathrm{Ga}$-Dotatoc showed $97 \%$ sensitivity, $92 \%$ specificity and $96 \%$ accuracy and had a major impact on management.

A study by Kayani et al ${ }^{14}$ of ${ }^{68} \mathrm{Ga}$-Dotatate and ${ }^{18} \mathrm{~F}$-FDG PET/CT in 38 NET patients found a higher sensitivity (88\%) for ${ }^{68} \mathrm{Ga}$-Dotatate, particularly in well- differentiated tumours, compared with ${ }^{18} \mathrm{~F}$ FDG PET/CT (66\%). Other PET radiopharmaceuticals were also found to be inferior to ${ }^{68} \mathrm{Ga}$-peptides. ${ }^{15}$

Ambrosini et al ${ }^{16}$ compared ${ }^{18} \mathrm{~F}-\mathrm{DOPA}$ and ${ }^{68} \mathrm{Ga}$-Dotanoc in the evaluation of NETs. ${ }^{68} \mathrm{Ga}$-Dotanoc was positive in all 13 patients while ${ }^{18} \mathrm{~F}$-DOPA was positive in only nine of 13 .

${ }^{68} \mathrm{Ga}$-peptides are also useful in neuroectodermal tumours, a group of related conditions that includes phaeochromocytoma and paraganglioma, which also demonstrate hSSTR on their cell membrane. a

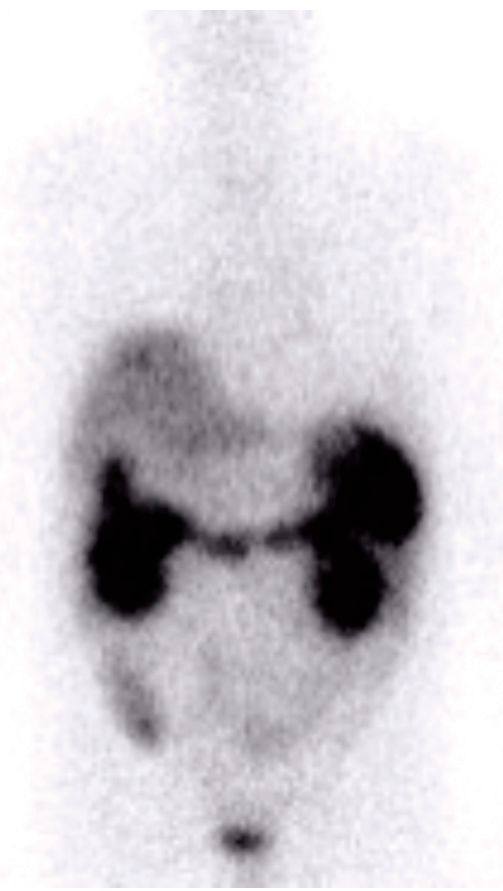

Results from our group showed a higher sensitivity compared with conventional ${ }^{123}$ I-mIBG, imaging, particularly in aggressive head and neck tumours. ${ }^{17,18}$

One of the interesting aspects of ${ }^{68} \mathrm{Ga}$-peptides PET/CT imaging is their ability to detect early bone involvement not discernible using CT or MRI (Fig 3). ${ }^{19,20}$ Early imaging with ${ }^{68} \mathrm{Ga}$-peptides could therefore have a significant positive impact on NET staging and consequent management.

The success of receptor imaging with radiolabelled peptides has paved the way
Fig 2. A 75-year-old female with carcinoid and liver metastases confirmed on CT (not shown): (a) Anterior image of ${ }^{111}$ In-octreotide shows equivocal lesion in the right lobe of the liver. Uptake noted in bowel loops is a normal variant. (b) Equivalent image of ${ }^{68} \mathrm{Ga}$-Dotatate shows two well-defined metastatic lesions in the liver. Bowel uptake is much less pronounced than in ${ }^{111}$ In-octreotide imaging.

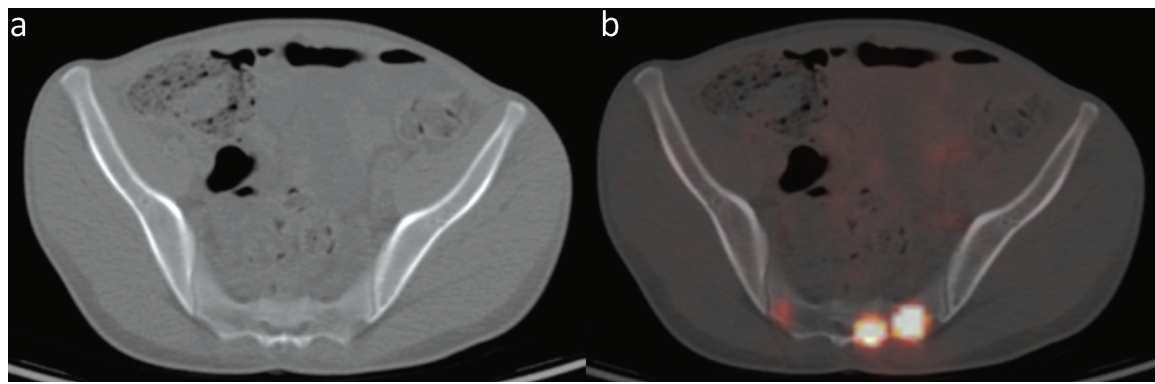

Fig 3. A 31-year-old male with metastatic neuroendocrine tumour: (a) CT shows normal texture and attenuation of pelvic bones. (b) Fused ${ }^{68} \mathrm{Ga}$-Dotatate PET/CT shows three bone metastases in the sacrum. 


\section{CME Nuclear medicine}

for further radionuclide imaging possibilities that are currently under development, targeting, for example, cholecystokinin, gastrin, glucagon and bombesin receptors.

\section{References}

1 Taal BG, Visser O. Epidemiology of neuroendocrine tumours. Neuroendocrinology 2004;80(Suppl 1):3-7.

2 Weckbecker G, Lewis I, Albert R et al. Opportunities in somatostatin research: biological, chemical and therapeutic aspects. Nat Rev Drug Discov 2003;2:999-1017.

3 Lamberts SW, Krenning EP, Reubi JC. The role of somatostatin and its analogs in the diagnosis and treatment of tumors. Review. Endocr Rev 1991;12:450-82.

4 Reubi JC, Schär JC, Waser B et al. Affinity profiles for human somatostatin receptor subtypes SST1-SST5 of somatostatin radiotracers selected for scintigraphic and radiotherapeutic use. Eur J Nucl Med 2000;27:273-82.

5 Krenning EP, Kwekkeboom DJ, Bakker WH et al. Somatostatin receptor scintigraphy with [111In-DTPA-D-Phe1]- and [123I-Tyr3]-octreotide: the Rotterdam experience with more than 1000 patients. Review. Eur J Nucl Med 1993;20:716-31.

6 Ezziddin S, Logvinski T, Yong-Hing C et al. Factors predicting tracer uptake in somatostatin receptor and MIBG scintigraphy of metastatic gastroenteropancreatic neuroendocrine tumors. J Nucl Med 2006;47:223-33.

7 Bombardieri E, Seregni E, Villano C et al. Position of nuclear medicine techniques in the diagnostic work-up of neuroendocrine tumors. Review. Q J Nucl Med Mol Imaging 2004;48:150-63.

8 Prasad V, Ambrosini V, Alavi A et al. PET/ CT in neuroendocrine tumors: evaluation of receptor status and metabolism. PET Clin 2007;2:351-75.

9 Hoegerle S, Altehoefer C, Ghanem N et al. Whole-body $18 \mathrm{~F}$ dopa PET for detection of gastrointestinal carcinoid tumors. Radiology 2001;220:373-80.

10 Orlefors H, Sundin A, Garske U et al. Whole-body (11)C-5-hydroxytryptophan positron emission tomography as a universal imaging technique for neuroendocrine tumors: comparison with somatostatin receptor scintigraphy and computed tomography. J Clin Endocrinol Metab 2005;90:3392-400.

11 Hofmann M, Maecke H, Börner R et al. Biokinetics and imaging with the somatostatin receptor PET radioligand (68) Ga-DOTATOC: preliminary data. Eur J Nucl Med 2001;28:1751-7.

12 Al-Nahhas A, Win Z, Szyszko T et al. Gallium-68 PET: a new frontier in receptor cancer imaging. Review. Anticancer Res 2007;27:4087-94.

13 Gabriel M, Decristoforo C, Kendler D et al. 68Ga-DOTA-Tyr3-octreotide PET in neuroendocrine tumors: comparison with somatostatin receptor scintigraphy and CT. J Nucl Med 2007;48:508-18.

14 Kayani I, Bomanji JB, Groves A et al. Functional imaging of neuroendocrine tumors with combined PET/CT using 68Ga-DOTATATE (DOTA-DPhe1,Tyr3octreotate) and 18F-FDG. Cancer 2008;112:2447-55.

15 Ambrosini V, Rubello D, Nanni C et al. 68Ga-DOTA-peptides versus 18F-DOPA
PET for the assessment of NET patients. Nucl Med Commun 2008;29:415-7.

16 Ambrosini V, Tomassetti P, Castellucci P et al. Comparison between 68Ga-DOTA-NOC and 18F-DOPA PET for the detection of gastro-entero-pancreatic and lung neuro-endocrine tumours. Eur J Nucl Med Mol Imaging 2008;35:1431-8.

17 Naji M, Zhao C, Welsh SJ et al. $68 \mathrm{Ga}-\mathrm{DOTA}-\mathrm{TATE}$ PET vs. $123 \mathrm{I}-\mathrm{MIBG}$ in identifying malignant neural crest tumours. Mol Imaging Biol 2011;13: 769-75.

18 Naji M, Al-Nahhas A. 68Ga-labelled peptides in the management of neuroectodermal tumours. Eur J Nucl Med Mol Imaging 2012;39(Suppl 1): S61-7.

19 Putzer D, Gabriel M, Henninger B et al. Bone metastases in patients with neuroendocrine tumour: ${ }^{68} \mathrm{Ga}$-DOTA-Tyr3octreotide PET in comparison to CT and bone scintigraphy. J Nucl Med 2009;50:1214-21.

20 Al-Nahhas A. Detection of unsuspected bone metastases by ${ }^{68} \mathrm{Ga}$-DOTA: nuclear medicine at the forefront again. $\mathrm{Nucl} \mathrm{Med}$ Commun 2011;32:877-9.

\section{Address for correspondence:}

Professor Adil Al-Nahhas,

Chief of Service of Nuclear Medicine, Hammersmith Hospital, Imperial College NHS Trust, Du Cane Road, London W12 0HS.

Email: adil.al-nahhas@imperial.nhs.uk 